\title{
Covariant Magnetic Connection Hypersurfaces
}

\author{
F. Pegoraro \\ Dipartimento di Fisica, Università di Pisa, Italy \\ francesco.pegoraro@unipi.it \\ Accepted for publication in Journal of Plasma Physics
}

\begin{abstract}
In the single fluid, nonrelativistic, ideal-Magnetohydrodynamic (MHD) plasma description magnetic field lines play a fundamental role by defining dynamically preserved "magnetic connections" between plasma elements. Here we show how the concept of magnetic connection needs to be generalized in the case of a relativistic MHD description where we require covariance under arbitrary Lorentz transformations. This is performed by defining 2-D magnetic connection hypersurfaces in the 4-D Minkowski space. This generalization accounts for the loss of simultaneity between spatially separated events in different frames and is expected to provide a powerful insight into the 4-D geometry of electromagnetic fields when $\mathbf{E} \cdot \mathbf{B}=0$.
\end{abstract}

Pacs: 52.27.Ny, 03.50.De, 52.35.Vd

\section{Introduction}

The dynamics of large scale relativistic plasma configurations plays an important role in our understanding of high energy astrophysical phenomena such as, just to mention a recently discovered one, the flaring of the Crab nebula [1]. Even without including general relativistic effects, as would be the case, e.g., in the neighbourhood of a black hole (see for example the system of equations investigated by [2]), the phenomena we need to describe involve velocities close to the speed of light and internal energies that can be larger than the electron rest mass energy. Furthermore relativistic plasmas with very large energy densities have been produced in the laboratory in laser plasma experiments and it has been stressed that such experiments can help us to understand high energy astrophysical phenomena (see e.g., [3]).

With this in mind, several concepts that have been introduced for nonrelativistic plasmas need to be extended to relativistic regimes. In such a generalization space and time properties are necessarily combined since the basic invariance properties of the matter equations are now given in terms of the Lorentz group of transformations between different reference frames. This is particularly important since, in the presence of very large velocity differences between different parts of the plasma configuration, there may not be a clear way of defining a preferred reference frame on physics grounds. In addition, the observer reference frame may move with a relativistic velocity with respect to the plasma under observation and thus observe as simultaneous events that are not simultaneous in the plasma frame. 
For phenomena occurring on macroscopic scales, i.e. on space and time scales large on the characteristic microscopic scales of the particle dynamics, the single fluid nonrelativistic MHD plasma description has been extended (see [4, 5]) and used in numerical simulations (see e.g., [6]) so as to include relativistic fluid velocities and relativistic internal energy densities. A Hamiltonian reformulation of ideal relativistic MHD dynamics in terms of noncanonical variables has been recently derived in [7]. In this process of generalization a number of basic phenomena of nonrelativistic MHD, such as e.g. magnetic reconnection, have been reconsidered in relativistic plasma regimes both in the laboratory (see [8, 9]), and in astrophysics (see [10, 11]). In particular in the astrophysical context relativistic magnetic reconnection has been considered mostly as a mechanism of energy conversion, usually choosing a preferred frame of reference, possibly thought of as an "average comoving frame", i.e. as a frame in which the plasma region under consideration is globally at rest. As mentioned above, such an approach may not be fully unambiguous in situations where very large velocity relativistic variations can be present between different plasma regions. This is so in particular from the observational point of view when describing magnetic reconnection structures since magnetic fields and electric fields are transformed one into the other when seen in a Lorentz boosted reference frame.

Thus an important point in the relativistic extension of the MHD plasma description is to provide a frame independent definition of magnetic reconnection. However such a definition is neither obvious from a theoretical nor from an observational point of view since, as already mentioned, the distinction between electric and magnetic fields is frame dependent and the tracing of field lines, which are only defined in coordinate space at fixed time, is also frame dependent due to the violation of simultaneity in different reference frames of events at different spatial locations.

Although a clearcut definition of magnetic reconnection is not simple to formulate even for a non relativistic plasma, its common definition is not simply limited to the fact that magnetic energy is converted to kinetic and/or internal plasma energy but refers to the local violation of the magnetic topology and, in particular, to the local breaking of the structure of magnetic connections.

Magnetic connections are defined by the fundamental property of ideal MHD (see [12]) that if two plasma elements, moving with plasma in a smooth flow, are connected at time $t$ by a magnetic field line then at any following time there exists a magnetic field line that connects them. This property is the conceptual basis from which the expressions that the magnetic field is frozen in the plasma and that field lines move with the plasma are derived.

Thus in order to define magnetic reconnection in a covariant way we must first obtain a covariant definition of magnetic connections. Again, such a definition is not a priori obvious because of two already mentioned related reasons: the distinction between electric and magnetic fields and the very concept of field lines are frame dependent. This point was explicitly addressed in [13] where it was shown that the covariant formulation of magnetic connections can be restored by means of a time resetting projection along the trajectories of the plasma elements. This projection is consistent with the ideal Ohm's law and compensates for the loss of simultaneity in different reference frames between spatially separated events.

In the present paper we address this same issue again and show that the time resetting along the trajectories of the fluid elements introduced in [13] is essentially equivalent to a redefinition of the geometrical object that we use in order to define magnetic connections. We argue that, while in 3-D (coordinate) space magnetic connections are defined by 1-D curves (field lines), in the 4-D Minkowski space they are defined by 2-D hypersurfaces that are generated by a suitably defined magnetic (space-like) 4-vector field and by the velocity (time-like) 4-vector field of the plasma. In fact, following a somewhat different line of approach from the one adopted in [13], we show 
that, if the electromagnetic (e.m) field tensor satisfies an ideal Ohm's law, it exhibits special geometrical properties that are simply the consequence of the homogeneous Maxwell's equations and that make it possible to define such 2-D hypersurfaces so that, if in a given frame two plasma elements in 4-D Minkowski space lie on the same 2-D hypersurface, they do so in any other reference frame.

We call these 2-D hypersurfaces (with one space-like and one time-like tangent vector field) Covariant Magnetic Connection Hypersurfaces, or connection hypersurfaces for short. The standard magnetic connections in 3-D space can then be recovered in any chosen reference frame by taking sections of these surfaces at a fixed (in that frame) time. We stress that these 2-D hypersurfaces bear no relation to the 3-D magnetic surfaces of nonrelativistic MHD that, if generalized to 4-D Minkowski space, would involve 3-D "volumes".

The present paper stops at this result, just after noting that the violation of the ideal Ohm's law leads to a violation of the geometrical properties of the e.m. field tensor that make it possible to define the connection hypersurfaces. Thus in this 4-D framework magnetic reconnection, caused by a local violation of the ideal Ohm's law, can be interpreted in a frame independent way as a local "piercing and merging" of connection hypersurfaces. These lose their identity only locally, in exactly the same way as magnetic field lines do in the standard 3-D space setting. The physical and observational consequences of this definition will be investigated in detail in a later paper.

However, even remaining within the validity of the ideal Ohm's law, i.e. without allowing for magnetic reconnection to occur, important open questions remain to be investigated: in particular how to generalize the study of the topological properties such as, e.g., field line braiding (see e.g., [14]), that have been investigated within a fixed frame 3-D description to the study of the properties of connection hypersurfaces in 4-D Minkowski space. In the present paper only some very general properties of the magnetic helicity 4-vector field are discussed and are shown to allow us to define a Lorentz-scalar Lagrangian invariant that is advected by the plasma motion.

Before entering the detailed derivation of the covariant connection hypersurfaces we stress that their definition only requires that an ideal Ohm's law be valid, supplemented by the homogeneous Maxwell's equations. The inhomogeneous Maxwell's equations, that relate the e.m. field tensor to the charge and current densities and that thus determine the field dynamics from the plasma dynamics, are not directly involved in the definition of the connection hypersurfaces which, in this sense, are more general than relativistic MHD and thus apply under more general conditions.. Depending on the plasma description adopted, the connection hypersurfaces can either relate to the single fluid description or to a selected species in the plasma, generally the lighter one. Physically, the main assumption that is made is that kinetic effects can be neglected in the chosen regime for this lighter species and that a fluid velocity can be defined, independently of whether it is a single fluid velocity, as in MHD, or e.g., the electron velocity. We also note (see e.g., [16]) that electron inertia effects and electron thermal effects (for an isotropic and isentropic thermodynamic closure) can be included by a suitable redefinition of the electromagnetic field tensor. In fact, this redefined field tensor obeys an ideal Ohm's law and a set of equations analogous in form to the homogeneous Maxwell equations. On the other hand dissipative effects, either resistive or arising from the "friction term" due to incoherent high frequency radiation in fully relativistic regimes, can lead to violation of the ideal Ohm's law, in particular in the presence of a nonlinear plasma dynamics that leads to the formation of smaller and smaller space and time scales. If these effects are local, they provide the local breaking and merging of the connection hypersurfaces involved in magnetic reconnection. Finally the case of an electron-positron plasma where there are two light species, and that is quite important for astrophysics, would require within the present framework the additional assumption that both species satisfy an ideal Ohms law (not necessarily the same). 


\section{Ideal Ohm's law}

An important feature of the ideal 3-D Ohm's law

$$
\mathbf{E}+\mathbf{v} \times \mathbf{B} / c=0, \quad \Rightarrow \mathbf{E} \cdot \mathbf{B}=0,
$$

with $\mathbf{v}$ the 3-D plasma fluid velocity field and $\mathbf{E}$ and $\mathbf{B}$ the electric and the magnetic fields, is that it is in no sense restricted to a nonrelativistic plasma regime or to a preferred reference frame. In fact it can be written (unmodified) in the fully covariant form (see e.g., [15])

$$
\mathbf{F}_{\mu \nu} \mathbf{u}^{\nu}=0
$$

where $\mathbf{F}_{\mu \nu}$ is the e.m. field tensor, $\mathbf{u}^{\mu}$ is a timelike 4-vector which we interpret as the fluid velocity 4-vector field of the plasma (or of the plasma species with respect to which the magnetic field is frozen, see e.g. the generalized formulation given in [16, 17, 18]).

From Eq. (1) and Faraday's equation $\nabla \times \mathbf{E}+(1 / c) \partial \mathbf{B} / \partial t=0$, the 3 -D magnetic equation

$$
\partial \mathbf{B} / \partial t-\nabla \times(\mathbf{v} \times \mathbf{B})=0
$$

follows, together with the 3-D connection theorem [12] mentioned in the Introduction: if at $t=0$ we have $d \mathbf{l} \times \mathbf{B}=0$, where $d \mathbf{l}$ is the the vector field tangent to a curve connecting two plasma elements, i.e., if the two elements are connected by a magnetic field line, then $d \mathbf{l} \times \mathbf{B}=0$ for all $t$ since

$$
\frac{d}{d t}(d \mathbf{l} \times \mathbf{B})=-(d \mathbf{l} \times \mathbf{B})(\nabla \cdot \mathbf{v})-[(d \mathbf{l} \times \mathbf{B}) \times \nabla] \mathbf{v} .
$$

Here $d / d t$ is the Lagrangian time derivative along the plasma element motion.

While the ideal Ohm's law (1) can be set in an explicitly covariant form (2), the interpretation of Eq.(4) in terms of the conservation of magnetic connections cannot be directly transferred to a different reference frame, as can be seen from the fact that a Lorentz boost will in general add a time component to the transformed vector field $d \mathbf{l}^{\prime}$ so that it will no longer be possible to interpret it as the vector field tangent to a curve in 3-D (coordinate) space.

However the simple fact that Ohm's law is fully covariant suggests that it must be possible to reformulate the connection theorem in a frame independent way.

\subsection{Lichnerowicz-Anile representation}

In contrast to [13], here we adopt the two 4-vector fields representation [4, 5, 7] of the e.m. field tensor $\mathbf{F}_{\mu \nu}$

$$
\mathbf{F}_{\mu \nu}=\varepsilon_{\mu \nu \lambda \sigma} \mathbf{b}^{\lambda} \mathbf{u}^{\sigma}+\left[\mathbf{u}_{\mu} \mathbf{e}_{\nu}-\mathbf{u}_{\nu} \mathbf{e}_{\mu}\right],
$$

where $\mathbf{b}^{\mu}$ is the 4-vector magnetic field and $\mathbf{e}_{\mu}$ is the 4-vector electric field, with $\mathbf{u}^{\mu} \mathbf{e}_{\mu}=0$ and $\mathbf{u}_{\mu} \mathbf{b}^{\mu}=0$. The 4-vectors $\mathbf{e}_{\mu}$ and $\mathbf{b}^{\mu}$ are related to the standard electric and magnetic fields $\mathbf{E}$ and B in 3-D space by

$$
\mathbf{b}^{\mu}=\gamma(\mathbf{B}+\mathbf{E} \times \mathbf{v}, \mathbf{B} \cdot \mathbf{v})
$$

and

$$
\mathbf{e}_{\mu}=\gamma(\mathbf{E}+\mathbf{v} \times \mathbf{B},-\mathbf{E} \cdot \mathbf{v}),
$$

with $\mathbf{e}_{\mu} \mathbf{b}^{\mu}=\mathbf{E} \cdot \mathbf{B}$. We have adopted the Minkowski metric tensor $\eta_{\mu \nu}$ defined by $(+,+,+,-)$ and normalized 3-D velocities $\mathbf{v}$ to the speed of light: $\gamma$ is the relativistic Lorentz factor and $\mathbf{u}^{\mu}=\gamma(\mathbf{v}, 1)$ and $\mathbf{u}_{\mu} \mathbf{u}^{\mu}=-1$. The orthogonality conditions $\mathbf{u}^{\mu} \mathbf{e}_{\mu}=\mathbf{u}_{\mu} \mathbf{b}^{\mu}=0$ make this 
representation unique. In the following we will call this representation the Lichnerowicz-Anile (LA) representation. The LA representation is physically convenient as it allows us to separate covariantly the magnetic and the electric parts of the e.m. field tensor on dynamical grounds, i.e. relative to the plasma velocity 4 -vector field $\mathbf{u}^{\mu}$. In the local rest frame of a plasma element the time components of $\mathbf{e}_{\mu}$ and of $\mathbf{b}^{\mu}$ vanish, while their space components reduce to the standard 3-D electric and magnetic fields.

A corresponding representation holds for the dual tensor $\mathbf{G}^{\mu \nu} \equiv \varepsilon^{\mu \nu \alpha \beta} \mathbf{F}_{\alpha \beta} / 2$ with $\mathbf{e}_{\mu}$ and $\mathbf{b}^{\mu}$ interchanged. Thus:

$$
\mathbf{G}^{\mu \nu}=\varepsilon^{\mu \nu \lambda \sigma} \mathbf{u}_{\lambda} \mathbf{e}_{\sigma}+\left[\mathbf{u}^{\mu} \mathbf{b}^{\nu}-\mathbf{u}^{\nu} \mathbf{b}^{\mu}\right], \quad \text { with } \mathbf{e}_{\mu}=\mathbf{F}_{\mu \nu} \mathbf{u}^{\nu} \quad \text { and } \quad \mathbf{b}^{\mu}=\mathbf{G}^{\mu \nu} \mathbf{u}_{\nu} .
$$

If the ideal Ohm's law $\mathbf{F}_{\mu \nu} \mathbf{u}^{\nu}=0$ holds, the electric 4-vector $\mathbf{e}_{\mu}$ vanishes, the tensors $\mathbf{F}_{\mu \nu}$ and $\mathrm{G}^{\mu \nu}$ have rank two and can be written as

$$
\mathbf{F}_{\mu \nu}=\varepsilon_{\mu \nu \lambda \sigma} \mathbf{b}^{\lambda} \mathbf{u}^{\sigma}, \quad \mathbf{G}^{\mu \nu}=\left[\mathbf{u}^{\mu} \mathbf{b}^{\nu}-\mathbf{u}^{\nu} \mathbf{b}^{\mu}\right]
$$

with

$$
\begin{gathered}
\mathbf{F}_{\mu \nu} \mathbf{b}^{\nu}=\mathbf{F}_{\mu \nu} \mathbf{u}^{\nu}=0, \\
\mathbf{F}_{\mu \nu} \mathbf{G}^{\nu \mu}=0 \rightarrow \mathbf{E} \cdot \mathbf{B}=0, \quad \text { and } \quad \mathbf{b}_{\mu} \mathbf{b}^{\mu}=\mathbf{G}_{\mu \nu} \mathbf{G}^{\nu \mu} / 2=\mathbf{F}_{\mu \nu} \mathbf{F}^{\nu \mu} / 2 .
\end{gathered}
$$

In this case we can use $\mathbf{e}_{\mu}=0$ in order to express $\mathbf{b}^{\mu}$ in terms of $\mathbf{B}$ and $\mathbf{v}$ only as

$$
\mathbf{b}^{\mu}=\gamma\left(\mathbf{B} / \gamma^{2}+\mathbf{v}(\mathbf{v} \cdot \mathbf{B}), \mathbf{B} \cdot \mathbf{v}\right) \text {. }
$$

Note that in general $\partial_{\mu} \mathbf{b}^{\mu} \neq 0$ while from Maxwell's equations we have

$$
\partial_{\mu} \mathbf{G}^{\mu \nu}=0
$$

and thus

$$
\partial_{\mu} \mathbf{b}^{\mu}=\mathbf{G}^{\mu \nu}\left(\partial_{\mu} \mathbf{u}_{\nu}\right)=\mathbf{b}^{\nu}\left(\partial_{\tau} \mathbf{u}_{\nu}\right)
$$

where $\partial_{\tau}=\mathbf{u}^{\mu} \partial_{\mu}$ with $\tau$ the proper time and $\partial_{\tau} \mathbf{u}_{\nu}$ the 4-acceleration of the plasma element. Unless explicitly stated in the rest of this article we will assume $\mathbf{e}_{\mu} \equiv 0$.

\section{Covariant magnetic 4-vector field equation}

From Eqs. (9) and (13) we obtain the "magnetic vector field equation"

$$
\mathbf{u}^{\mu} \partial_{\mu} \mathbf{b}^{\nu}-\mathbf{b}^{\mu} \partial_{\mu} \mathbf{u}^{\nu}+\mathbf{b}^{\nu} \partial_{\mu} \mathbf{u}^{\mu}-\mathbf{u}^{\nu} \partial_{\mu} \mathbf{b}^{\mu}=0
$$

i.e.

$$
\partial_{\tau} \mathbf{b}^{\nu}=\mathbf{u}^{\nu} \partial_{\mu} \mathbf{b}^{\mu}-\mathbf{b}^{\nu} \partial_{\mu} \mathbf{u}^{\mu}+\mathbf{b}^{\mu} \partial_{\mu} \mathbf{u}^{\nu}
$$

which differs "in form" from the standard 3-D magnetic field equation, as obtained e.g. from Eq.(3) by expanding the $\nabla \times(\mathbf{v} \times \mathbf{B})$ term, because $\mathbf{b}^{\mu}$ is not divergence-free. Inserting Eq.(14) into Eq.(16) we obtain the magnetic equation in relativistic Lagrangian variables

$$
\partial_{\tau} \mathbf{b}^{\nu}=\mathbf{u}^{\nu} \mathbf{b}^{\alpha}\left(\partial_{\tau} \mathbf{u}_{\alpha}\right)-\mathbf{b}^{\nu} \partial_{\mu} \mathbf{u}^{\mu}+\mathbf{b}^{\mu} \partial_{\mu} \mathbf{u}^{\nu}
$$

where only 3 components are independent. Eq.(17) can also be written in projection form as

$$
\left(\delta_{\alpha}^{\nu}+\mathbf{u}^{\nu} \mathbf{u}_{\alpha}\right) \partial_{\tau} \mathbf{b}^{\alpha}=\mathbf{b}^{\mu} \partial_{\mu} \mathbf{u}^{\nu}-\mathbf{b}^{\nu} \partial_{\mu} \mathbf{u}^{\mu}
$$




\subsection{Frobenius condition and 2-D Hypersurfaces}

Equation (15) can be viewed as a Frobenius involution condition for the 4-vector fields $\mathbf{b}^{\mu}$ and $\mathbf{u}^{\mu}$. This condition, which is a consequence of the homogeneous Maxwell's equations $\partial_{\mu} \mathbf{G}^{\mu \nu}=0$, i.e. of Faraday's equation and of B being divergence-free, and of the ideal Ohm's law, allows us 1 to construct in the 4-D space-time 2-D hypersurfaces generated by the vector fields $\mathbf{u}^{\mu}$ and $\mathbf{b}^{\mu}$.

These hypersurfaces, which we call connection hypersurfaces because they will allow us to recast the connection theorem (4) in a covariant form, see Sec.5, are the 4-D counterpart of magnetic field lines in 3-D space and are not related to the magnetic surfaces defined in 3-D by the equation B $\cdot \nabla \psi=0$.

\section{Gauge freedom in the LA representation}

We can assume, without loss of generality, that the velocity 4-vector $\mathbf{u}^{\mu}$ satisfies a continuity equation of the form

$$
\partial_{\mu}\left(n \mathbf{u}^{\mu}\right)=0
$$

where $n$ can be taken to play the role of the proper density of the plasma element and $n \mathbf{u}^{\mu}$ of the density 4-vector.

As shown in detail in [7] a gauge freedom is allowed in the definition of the magnetic 4-vector field $\mathbf{b}^{\mu}$ in the LA representation provided we relax the orthogonality condition $\mathbf{b}^{\mu} \mathbf{u}_{\mu}=0$ :

$$
\mathbf{b}^{\mu} \rightarrow \mathbf{h}^{\mu} \equiv \mathbf{b}^{\mu}+g \mathbf{u}^{\mu}
$$

where $g$ is a free scalar field. Different choices of the gauge field $g$ allow us to impose specific conditions on $\mathbf{h}^{\mu}$. If, as in [7], we choose the divergence gauge

$$
\partial_{\tau}(g / n)=-(1 / n) \partial_{\mu} \mathbf{b}^{\mu}
$$

we have $\partial_{\mu} \mathbf{h}^{\mu}=0$, while, if we take in a given frame 2 the magnetic gauge

$$
g=-\mathbf{v} \cdot \mathbf{B}
$$

we can make the time component of $\mathbf{h}^{\mu}$ vanish and $\mathbf{h} \| \mathbf{B}$ in that frame.

Note that, since the expression for $\mathbf{G}^{\mu \nu}$ is unchanged if we insert $\mathbf{h}^{\mu}$ for $\mathbf{b}^{\mu}$ in Eq. (9), the Frobenius condition (15) holds independently of the gauge. Thus the connection-hypersurfaces generated by the 4-vector fields $\mathbf{u}^{\mu}$ and $\mathbf{b}^{\mu}$ can also be seen as generated by the 4-vector fields $\mathbf{u}^{\mu}$ and $\mathbf{h}^{\mu}$. For the sake of notational clarity in the following we will denote by $\mathbf{h}_{\|}^{\mu}$ the 4-vector field corresponding to the magnetic gauge (22) and specifically by $\mathbf{h}^{\mu}$ without any additional mark the 4-vector field corresponding to the divergence gauge (21).

\section{Covariant Connection theorem}

Extending the procedure developed in Sec.2 to 4-D Minkowski space, we consider in a given frame a magnetic field line $\ell$ at a fixed time in 4-D Minkowski space with tangent (spacelike) 4-vector

\footnotetext{
${ }^{1}$ Provided $\mathbf{b}^{\mu} \neq 0$, see Sec7

${ }^{2}$ The quantity $-\mathbf{v} \cdot \mathbf{B}$ is a Lorentz scalar. Its expression in a frame moving with respect to the chosen frame with velocity 4-vector $V_{\mu}$ is $-\left(V_{\mu} b^{\mu}\right) /\left(V_{\nu} u^{\nu}\right)$.
} 
field $d \mathbf{l}^{\mu}$. In this frame its time component $d l^{o}=0$ and the condition $\mathbf{F}_{\mu \nu} d \mathbf{l}^{\nu}=0$ implies $d \mathbf{l} \times \mathbf{B}=0$ corresponding 3 to the field line condition used in Sec, 2 .

Recalling that the rank of $\mathbf{F}_{\mu \nu}$ must be even, the condition $\mathbf{F}_{\mu \nu} d \mathbf{l}^{\nu}=0$ also implies that $d \mathbf{l}^{\mu}$ must be a linear combination of $\mathbf{b}^{\mu}$ and $\mathbf{u}^{\mu}$ (aside for the null points of $\mathbf{F}_{\mu \nu}$, see Sec.7) i.e. that it lies on a connection hypersurface defined in Sec.3.1

\subsection{Time resetting gauge}

The condition $\mathbf{F}_{\mu \nu} d \mathbf{l}^{\nu}=0$ remains valid even without imposing $d l^{\circ}=0$ because of the "time gauge" freedom $d \mathbf{l}^{\mu} \rightarrow d \hat{\mathbf{l}}^{\mu}=d \mathbf{l}^{\mu}+\mathbf{u}^{\mu} d \lambda$, with $\lambda$ a scalar function, i.e. $d \hat{\mathbf{l}}^{\mu}$ remains in the hypersurface generated by $\mathbf{b}^{\mu}$ and $\mathbf{u}^{\mu}$.

Conversely, in a boosted frame (where quantities are denoted by a "prime") the transformed vector field $d \mathbf{l}^{\prime \mu}$ will acquire a time component but will still lie on the boosted 2-D hypersurface generated by the boosted vector fields $\mathbf{b}^{\prime \mu}$ and $\mathbf{u}^{\prime \mu}$. Then, using the time gauge in reverse as done in [13], it will be possible to set $d l^{\prime o}=0$ without violating the condition in the boosted frame $\mathbf{F}_{\mu \nu}^{\prime} d \mathbf{l}^{\prime \nu}=0$ because of the ideal Ohm's law.

\subsection{Magnetic gauge}

After performing the time resetting gauge, using the magnetic gauge given by Eq. (22) we can bring the boosted 4-vector field $\mathbf{b}^{\prime \mu}$ to the form $\mathbf{h}_{\|}^{\prime \mu}=\left(0, \mathbf{B}^{\prime} / \gamma\right)$. Then in the boosted frame $\mathbf{F}_{\mu \nu}^{\prime} d \mathbf{l}^{\prime \nu}=0$ implies $\mathbf{d l}^{\prime} \times \mathbf{B}^{\prime}=0$.

This proves that it is possible to define magnetic connections in a covariant way, provided we refer to connection hypersurfaces instead of connection field lines and provided we properly "gauge" the 4-vector magnetic field $\mathbf{b}^{\mu}$ and the tangent (spacelike) 4-vector field $d \mathbf{l}^{\mu}$ within the connection hypersurface in order to compensate for the mixing between 3-D magnetic and electric fields under a Lorentz boost and for the loss of simultaneity in different frames.

Magnetic connections in 3-D space can then be recovered in any chosen reference frame by taking sections of these surfaces at a fixed (in that frame) time.

\section{Coordinates on Connection Hypersurfaces}

Choosing instead the divergence gauge (21), the Frobenius condition (15) can be reformulated in a way that allows us to define the following two commuting operators

$$
\partial_{\tau}=\left(\mathbf{n}^{\mu} / n\right) \partial_{\mu} \quad \text { and } \quad \partial_{h}=\left(\mathbf{h}^{\mu} / n\right) \partial_{\mu},
$$

where

$$
\begin{aligned}
& \partial_{\tau} \partial_{h}-\partial_{h} \partial_{\tau}=\left[\left(\mathbf{n}^{\mu} / n\right) \partial_{\mu}\left(\mathbf{h}^{\nu} / n\right)-\left(\mathbf{h}^{\mu} / n\right) \partial_{\mu}\left(\mathbf{n}^{\nu} / n\right)\right] \partial_{\nu} \\
= & (1 / n)\left[\partial_{\mu}\left(\mathbf{n}^{\mu} \mathbf{h}^{\nu} / n-\mathbf{h}^{\mu} \mathbf{n}^{\nu} / n\right)\right] \partial_{\nu}=(1 / n)\left[\partial_{\mu} \mathbf{G}^{\mu \nu}\right] \partial_{\nu}=0 .
\end{aligned}
$$

Then the set of curves on a Connection Hypersurface with tangent fields $\mathbf{n}^{\mu} / n=\mathbf{u}^{\mu}$ and $\mathbf{h}^{\mu} / n$ define a (nonorthogonal, and in general only local) coordinate system on the connection hypersurface. From the Minkowski line element $d s^{2}=d \mathbf{x}^{\mu} \eta_{\mu \nu} d \mathbf{x}^{\nu}$ we obtain the following expression for line element on a connection hypersurface

$$
d s^{2}=-d \tau^{2}+\left(\mathbf{h}_{\mu} \mathbf{h}^{\mu} / n^{2}\right) d h^{2}-(2 g / n) d h d \tau,
$$

\footnotetext{
${ }^{3}$ It includes $d \mathbf{l} \cdot \mathbf{E}=0$ which is satisfied if the ideal Ohm law holds
} 
where $\mathbf{h}_{\mu} \mathbf{h}^{\mu}=\mathbf{b}_{\mu} \mathbf{b}^{\mu}-g^{2}$ and the gauge function $g$ is defined by Eq.(21).

\section{Advected magnetic 4-D Nulls}

When using the LA representation to construct the connection-hypersurfaces we have not considered the null points of the e.m. tensor $\mathbf{F}_{\mu \nu}$ explicitly. Note that at these 4-D null points both the magnetic and the electric field vanish, which is a frame independent condition.

Since the velocity 4-vector $\mathbf{u}^{\mu}$ has no nulls $\left(\mathbf{u}^{\mu} \mathbf{u}_{\mu}=-1\right)$, in the LA representation with $\mathbf{e}_{\mu}=0$ a 4-D null of $\mathbf{F}_{\mu \nu}$ implies a 4-D null of $\mathbf{b}^{\mu}$ and viceversa a null of $\mathbf{b}^{\mu}$ implies 4 a null of $\mathbf{B}$.

A generic local expansion around a null 4-point (placed at the origin of the coordinate system) truncated at the first term reads

$$
\mathbf{b}^{\mu}=N_{\nu}^{\mu} \mathbf{x}^{\nu}, \quad \text { where } \quad \mathbf{u}_{\mu} \mathbf{b}^{\mu}=0 \Rightarrow \mathbf{u}_{\mu} N_{\nu}^{\mu}=0 .
$$

Here $N_{\nu}^{\mu}$ is a numerical tensor that in general need not be symmetric5. Then

$$
\mathbf{G}^{\mu \nu}=\left(N_{\alpha}^{\nu} \mathbf{u}^{\mu}-N_{\alpha}^{\mu} \mathbf{u}^{\nu}\right) \mathbf{x}^{\alpha} \quad \text { and } \quad \partial_{\mu} \mathbf{G}^{\mu \nu}=0 \Rightarrow N_{\mu}^{\nu} \mathbf{u}^{\mu}-N_{\mu}^{\mu} \mathbf{u}^{\nu}=0
$$

at the null. Contracting the latter identity with $\mathbf{u}_{\nu}$ and using $\mathbf{u}_{\nu} N_{\mu}^{\nu}=0$ from the r.h.s. of Eq.(26), we find at the 4-D null $N_{\mu}^{\mu}=0$, i.e. $\partial_{\mu} \mathbf{b}^{\mu}=0$ at the null and $N_{\mu}^{\nu} \mathbf{u}^{\mu}=0$. Thus in the instantaneous local rest frame of the 4-D null $N_{\nu}^{\mu}$ reduces to a 3-D tensor (only its space-space components do not vanish).

Finally we note that, since $\partial_{\mu} \mathbf{b}^{\mu}=0$ at the null point, from Eq.(16) we find that at the null point $\partial_{\tau} \mathbf{b}^{\mu}=0$, which can be used to trace along the fluid element trajectory the singularities of the connection-hypersurfaces that arise at the nulls of $\mathbf{b}^{\mu}$.

Furthermore, because of the two above conditions at the null point, we can take the gauge function $g$ in Eq. (21) equal to zero at the null point so that a null of $\mathbf{b}^{\mu}$ corresponds to a null of $\mathbf{h}^{\mu}$. Conversely, since $\mathbf{b}^{\mu}$ cannot be equal to $g \mathbf{u}^{\mu}$ with $g \neq 0$ because of the orthogonality condition $\mathbf{u}_{\mu} \mathbf{b}^{\mu}=0$, a null point of $\mathbf{h}^{\mu}$ must correspond to a null point of $\mathbf{b}^{\mu}$ and of $g$.

\section{Covariant Magnetic Helicity}

As is well known, the homogeneous Maxwell equation $\partial_{\mu} \mathbf{G}^{\mu \nu}=0$ implies that we can introduce a 4-vector potential field $\mathbf{A}_{\mu}$ such that $\mathbf{F}_{\mu \nu}=\partial_{\mu} \mathbf{A}_{\nu}-\partial_{\nu} \mathbf{A}_{\mu}$. The introduction of the vector potential allows us for a general e.m. field to give a covariant definition of the 4-vector magnetic helicity in the form

$$
\mathbf{K}^{\mu} \equiv \mathbf{G}^{\mu \nu} \mathbf{A}_{\nu}
$$

such that

$$
\partial_{\mu} \mathbf{K}^{\mu}=-\mathbf{F}_{\mu \nu} \mathbf{G}^{\nu \mu} / 2 .
$$

The magnetic helicity 4-vector is defined up to a 4-divergence $\partial_{\nu}\left(\chi \mathbf{G}^{\mu \nu}\right)$, with $\chi$ a scalar field, because of the usual gauge freedom in the definition of the 4-vector potential $\mathbf{A}_{\nu} \rightarrow \mathbf{A}_{\nu}+\partial_{\nu} \chi$. The r.h.s. of Eq.(29) vanishes if the ideal Ohm's law holds. In this case from Eqs.(9) we find

$$
\mathbf{K}^{\mu}=\mathbf{u}^{\mu}\left(\mathbf{b}^{\nu} \mathbf{A}_{\nu}\right)-\mathbf{b}^{\mu}\left(\mathbf{u}^{\nu} \mathbf{A}_{\nu}\right),
$$

\footnotetext{
${ }^{4}$ Such a one to one relationship is not generally true in the case where $\mathbf{e}_{\mu} \neq 0$ where, e.g., a null of $\mathbf{B}$ at the $X$-point of a reconnecting magnetic field does not imply a null of $\mathbf{b}_{\mu}$.

${ }^{5}$ Its antisymmetric part is related to the current density 4-vector at the null point
} 
i.e. $\mathbf{K}^{\mu}$ lies on connection-hypersurfaces. From the conditions $\mathbf{F}_{\mu \nu} \mathbf{u}^{\nu}=0$ and $\mathbf{F}_{\mu \nu} \mathbf{b}^{\nu}=0$, we obtain

$$
\mathbf{u}^{\mu} \partial_{\mu} \mathbf{A}_{\nu}=\mathbf{u}^{\mu} \partial_{\nu} \mathbf{A}_{\mu}, \quad \mathbf{b}^{\mu} \partial_{\mu} \mathbf{A}_{\nu}=\mathbf{b}^{\mu} \partial_{\nu} \mathbf{A}_{\mu},
$$

and, using $\partial_{\mu} \mathbf{G}^{\mu \nu}=0$ and Eq.(31), we verify that

$$
\partial_{\mu} \mathbf{K}^{\mu}=\mathbf{G}^{\mu \nu} \partial_{\mu} \mathbf{A}_{\nu}=\left(\mathbf{u}^{\mu} \mathbf{b}^{\nu}-\mathbf{b}^{\mu} \mathbf{u}^{\nu}\right)\left(\partial_{\mu} \mathbf{A}_{\nu}\right)=\mathbf{b}^{\mu} \mathbf{u}^{\nu} \partial_{\mu} \mathbf{A}_{\nu}-\mathbf{b}^{\nu} \mathbf{u}^{\mu} \partial_{\nu} \mathbf{A}_{\mu} \equiv 0
$$

If we choose the gauge scalar function $\chi$ such that $\mathbf{A}_{\mu} \mathbf{u}^{\mu}=0$, i.e. if we make the time component of the 4-vector potential vanish in the local rest frame, from Eqs.(30), (32) and (19) we obtain

$$
\partial_{\mu}\left[\mathbf{n}^{\mu}\left(\mathbf{b}^{\nu} \mathbf{A}_{\nu}\right) / n\right]=\mathbf{n}^{\mu} \partial_{\mu}\left(\mathbf{b}^{\nu} \mathbf{A}_{\nu} / n\right)=0 \quad \rightarrow \quad \partial_{\tau}\left(\mathbf{b}^{\nu} \mathbf{A}_{\nu} / n\right)=0,
$$

which provides us with a Lagrangian invariant scalar field advected by the plasma flow.

\section{Conclusions}

In this article we have addressed the problem of defining covariant magnetic connections for a relativistic plasma that obeys the ideal Ohm's law and have obtained the following main results.

1) We have reformulated the covariant connection theorem discussed in [13] in terms of 2-D hypersurfaces in 4-D Minkowski space making use of

a) the representation of the electromagnetic field tensor in terms of two 4-vector fields (which we called the Lichnerowicz-Anile representation) in the case where the ideal Ohm's law holds,

b) the gauge freedom in the definition of the magnetic 4-vector field,

c) a time-gauge transformation (time resetting) of the 4-vector field tangent to the curve connecting two plasma elements in 4-D space.

We call these hypersurfaces connection hypersurfaces.

2) We have indicated that these connection hypersurfaces take the role, for the full electromagnetic field tensor in 4-D, of the magnetic field lines in 3-D.

We thus argue that a covariant definition of magnetic reconnection may be given in a 4-D framework as a local "piercing and merging" of connection hypersurfaces that lose their identity only locally (where $\mathbf{e}_{\mu} \neq 0$ and the Frobenius condition does not hold), just as magnetic field lines do in the standard 3-D space setting.

Regarding point 1), we stress that different forms of gauge freedom play a very important role in our formulation, a common feature of electrodynamic theory. In fact the use of gauge transformations is a convenient tool for implementing useful but non-explicitly covariant conditions in a covariant theory. A well known example is provided by the transverse potential gauge condition ( $\phi=0$, with $\phi$ the time component of the vector potential) for a plane electromagnetic wave. This condition is not explicitly covariant, i.e. it is not in general preserved by a Lorentz boost, but can be restored by a gauge transformation of the boosted vector potential.

Point 2) suggests that the investigation of topological properties of the magnetic field in 3-D space, which play a fundamental role in ideal MHD, should be extended to the investigation of the topological properties of the full electromagnetic field tensor in 4-D space. This future line of enquiry may well open a novel and rich way of reinterpreting the topological properties of ideal MHD.

Finally we note again that the treatment developed in the present paper does not involve the full set of MHD plasma equations and only requires that an ideal Ohm's law in terms of a fluid velocity 
be satisfied. Thus this treatment can be applied to different plasma theories where the velocity 4-vector field $\mathbf{u}^{\mu}$ is not the plasma fluid velocity but, for example, the electron fluid velocity as is the case, e.g., in EMHD (see [19]).

\section{Acknowledgments}

The author acknowledges clarifying discussions with G. Tomassini, P.J. Morrison and E. D’Avignon.

\section{References}

[1] M. Tavani, et al., Science, 331, 736 (2011),

[2] S.Koide, Ap.J. , 708, 1459 (2010).

[3] S.V. Bulanov, T.Zh. Esirkepov, D. Habs, F. Pegoraro, T. Tajima, Eur. Phys. J. D 55, 483 (2009).

[4] A. Lichnerowicz, in Relativistic Hydrodynamics and Magnetohydrodynamics, (New York: Benjamin) 1967.

[5] M. Anile, in Relativistic fluids and magneto-fluids, Cambridge Monographs on Mathematical Physics, Cambridge 1989.

[6] A. Mignone, G. Bodo, Mon. Not. R. Astron. Soc., 368, 1040 (2006).

[7] E. D’Avignon, P.J. Morrison, F. Pegoraro, Phys. Rev. D, 91, 084050 (2015).

[8] G.A. Askaryan, S.V. Bulanov, F. Pegoraro, A.M. Pukhov, Comm. Plasma Phys. and Contr. Fusi., 17, 35 (1995).

[9] P.M. Nilson, et al., Phys. Rev. Lett., 97, 255001(2006).

[10] M. Hesse, S. Zenitani, Phys. Plasmas, 14, 112102 (2007).

[11] S. Zenitani, M. Hoshino, Astroph. J., 670, 702 (2007).

[12] W.A. Newcomb, Ann. Phys., 3, 347 (1958).

[13] F. Pegoraro, EPL, 99, 35001 (2012).

[14] M.A. Berger, Phys. Rev. Lett. , 70, 705 (1993).

[15] M. Gedalin, Phys. Rev. Lett., 76, 3340 (1996).

[16] F. Pegoraro, Phys. Plasmas, 22, 112106 (2015).

[17] F.A. Asenjo, L. Comisso, Phys. Rev. Lett., 114, 115003 (2015).

[18] F.A. Asenjo, L. Comisso, S.M. Mahajan Phys. Plasmas, 22, 122109 (2015).

[19] S.V. Bulanov, F. Pegoraro, A.S. Sakharov, Phys. Fluids B, 4, 2499 (1992). 